\title{
Augmented Arnoldi-Tikhonov Regularization Methods for Solving Large-Scale Linear Ill-Posed Systems
}

\author{
Yiqin Lin, ${ }^{1}$ Liang Bao, ${ }^{2}$ and Yanhua $\mathrm{Cao}^{3}$ \\ ${ }^{1}$ Department of Mathematics and Computational Science, Hunan University of Science and Engineering, Yongzhou 425100, China \\ ${ }^{2}$ Department of Mathematics, East China University of Science and Technology, Shanghai 200237, China \\ ${ }^{3}$ Department of Mathematics, North China Electric Power University, Beijing 102206, China
}

Correspondence should be addressed to Liang Bao; nlbao@yahoo.cn

Received 1 November 2012; Revised 18 March 2013; Accepted 19 March 2013

Academic Editor: Hung Nguyen-Xuan

Copyright (c) 2013 Yiqin Lin et al. This is an open access article distributed under the Creative Commons Attribution License, which permits unrestricted use, distribution, and reproduction in any medium, provided the original work is properly cited.

\begin{abstract}
We propose an augmented Arnoldi-Tikhonov regularization method for the solution of large-scale linear ill-posed systems. This method augments the Krylov subspace by a user-supplied low-dimensional subspace, which contains a rough approximation of the desired solution. The augmentation is implemented by a modified Arnoldi process. Some useful results are also presented. Numerical experiments illustrate that the augmented method outperforms the corresponding method without augmentation on some real-world examples.
\end{abstract}

\section{Introduction}

We consider the iterative solution of a large system of linear equations

$$
A x=b,
$$

where $A \in \mathbb{R}^{n \times n}$ is nonsymmetric and nonsingular, and $b \in \mathbb{R}^{n}$. We further assume that the coefficient matrix $A$ is of ill-determined rank; that is, all its singular values decay gradually to zero, with no gap anywhere in the spectrum. Such systems are often referred to as linear discrete illposed problems and arise from the discretization of ill-posed problems such as Fredholm integral equations of the first kind with a smooth kernel. The right-hand side $b$ of (1) is assumed to be contaminated by an error $e \in \mathbb{R}^{n}$, which may stem from discretization or measurement inaccuracies. Thus, $b=\widehat{b}+e$, where $\widehat{b}$ is the unknown error-free right-hand side vector.

We would like to compute the solution $\hat{x}$ of the linear system of equations with the error-free right-hand side $\widehat{b}$,

$$
A \widehat{x}=\widehat{b} \text {. }
$$

However, since the right-hand side in (2) is not available, we seek to determine an approximation of $\hat{x}$ by solving the available system (1) or a modification. Due to the ill conditioning of $A$, the system (1) has to be regularized in order to compute a useful approximation of $\hat{x}$. Perhaps the best known regularization method is Tikhonov regularization [13 ], which in its simplest form is based on the minimization problem

$$
\min _{x \in \mathbb{R}^{n}}\left\{\|A x-b\|^{2}+\frac{1}{\mu}\|x\|^{2}\right\},
$$

where $\mu>0$ is a regularization parameter. Here and throughout this paper $\|$. $\|$ denotes the Euclidean vector norm or the associated induced matrix norm.

After regulating the system (1), we need to compute the solution $x_{\mu}$ of the minimization problem (3). Such a vector $x_{\mu}$ is also the solution of

$$
\left(A^{T} A+\frac{1}{\mu} I\right) x=A^{T} b .
$$

Here and in the following, $I$ denotes the identity matrix, whose dimension is conformed with the dimension used in the context. If $\mu$ is far away from zero, then, due to the ill conditioning of $A, x_{\mu}$ is badly computed while, if $\mu$ is close to zero, $x_{\mu}$ is well computed, but the error $x_{\mu}-\widehat{x}$ is 
quite large. Thus, the choice of a good value for $\mu$ is fairly important. Several methods have been proposed to obtain an effective value for $\mu$. For example, if the norm of the error $e$ or a fairly accurate estimate is known, the regularization parameter is quite easy to determine by application of the discrepancy principle. The discrepancy principle proposes that the regularization parameter $\mu$ can be chosen so that the discrepancy $b-A x_{\mu}$ satisfies

$$
\left\|b-A x_{\mu}\right\|=\eta \varepsilon
$$

where $\varepsilon=\|e\|$ and $\eta>1$ is a constant; see, for example, [4] for further details on the discrepancy principle.

The singular value decomposition [5] of $A$ can be used to determine the solution $x_{\mu}$ of the minimization problem (3). For an overview of numerical methods for computing the SVD, we refer to [6]. We remark that the computational effort required to compute the SVD is quite high even for moderately sized matrices.

Many numerical methods using Krylov subspaces have been proposed for the solution of large-scale Tikhonov regularization problems (3). The main idea of such algorithms has been to first project the large problems onto some Krylov subspace to produce problems with small size and then solve the small-sized problems by the SVD. For instance, several well-established methods based on the Lanczos bidiagonalization process have been proposed for the solution of the minimization problem (3); see [7-10] and references therein. These methods use the Lanczos bidiagonalization process to construct a basis of the Krylov subspace:

$$
\begin{aligned}
\mathscr{K}_{m} & \left(A^{T} A, A^{T} b\right) \\
& =\operatorname{span}\left\{A^{T} b, A^{T} A A^{T} b, \ldots,\left(A^{T} A\right)^{m-1} A^{T} b\right\} .
\end{aligned}
$$

We remark that each Lanczos bidiagonalization step needs two matrix-vector product evaluations, one with $A$ and the other with $A^{T}$. Other methods using the Krylov subspace

$$
\mathscr{K}_{m}(A, A b)=\operatorname{span}\left\{A b, A^{2} b, \ldots, A^{m} b\right\}
$$

as the projection subspace have been also designed. For example, Lewis and Reichel [11] proposed to exploit the Arnoldi process to produce a basis of the Krylov subspace $\mathscr{K}_{m}(A, A b)$ to obtain an approximation of the solution of the Tikhonov regularization problem (3). Since each Arnoldi decomposition step requires only one matrix-vector evaluation with $A$, the approach based on the Arnoldi process may require fewer matrix-vector product evaluations than that based on the Lanczos bidiagonalization process. Moreover, the methods based on the Arnoldi process do not require the adjoint matrix $A^{T}$ and, hence, are more appropriate to problems for which the adjoint is difficult to evaluate. For such problems we refer to [12]. A similar Tikhonov regularization method based on generalized Krylov subspace is proposed in [13].
Some numerical methods without using the Tikhonov regularization technique have been already proposed to solve the large-scale linear discrete ill-posed problem (1). These methods include the range-restricted GMRES (RRGMRES) method [14, 15], the augmented range-restricted GMRES (ARRGMRES) method [16], and the flexible GMRES (FGMRES) method [17]. The RRGMRES method determines the $m$ th approximation $x_{m}$ of (1) by solving the minimization problem

$$
\min _{x \in \mathscr{K}_{m}(A, A b)}\|A x-b\|, \quad m=1,2, \ldots, x_{0}=0 .
$$

The regularization is implemented by choosing a suitable dimension number $m$; see, for example, [18]. The ARRGMRES method augments the Krylov subspace $\mathscr{K}_{m}(A, A b)$ by a low-dimensional user-supplied subspace. The lowdimensional subspace is determined by vectors that are able to represent the known features of the desired solution. The augmented method can yield approximate solutions of higher accuracy than the RRGMRES method if the Krylov subspace $\mathscr{K}_{m}(A, A b)$ does not allow representation of the known features.

In this paper, we propose a new iterative method, named augmented Arnoldi-Tikhonov regularization method, for solving large-scale linear ill-posed systems (1). The new method is deduced by combining the Tikhonov regularization technique and the augmentation technique. The augmentation is implemented by a modified Arnoldi process.

The following summarizes the structure of this paper. Section 2 describes the augmented Arnoldi-Tikhonov regularization method and some useful results. Some real-world examples are presented in Section 3. Section 4 contains the conclusions.

\section{Augmented Arnoldi-Tikhonov Regularization Method}

We attempt to improve the Arnoldi-Tikhonov regularization method proposed in [11] by augmenting the Krylov subspace $\mathscr{K}_{m}(A, A b)$ by a $k$-dimensional subspace $\mathscr{W}$, which contains a rough approximation of the desired solution of (2). Then, the subspace of projection we will exploit in the following is of the form

$$
\mathscr{K}_{m}(A, A b)+\mathscr{W}=\operatorname{span}\left\{A b, A^{2} b, \ldots, A^{m} b\right\}+\mathscr{W} .
$$

Let $W$ be the $n$ by $k$ matrix whose columns form an orthonormal basis of the space $\mathscr{W}$. For the purpose of augmentation by $\mathscr{W}$, we apply the modified Arnoldi process [16] to construct the modified Arnoldi decomposition

$$
A\left[W, V_{m}\right]=\left[U, V_{m+1}\right] H,
$$

where $U \in \mathbb{R}^{n \times k}, V_{m+1} \in \mathbb{R}^{n \times(m+1)},\left[U, V_{m+1}\right]$ has orthonormal columns, and $H \in \mathbb{R}^{(m+k+1) \times(m+k)}$ is an upper Hessenberg matrix. We point out that the leading principal $k$ by $k$ submatrix of $H$ is the upper triangular matrix in the QR factorization [5] of $A W$; that is, $H$ is of the form 


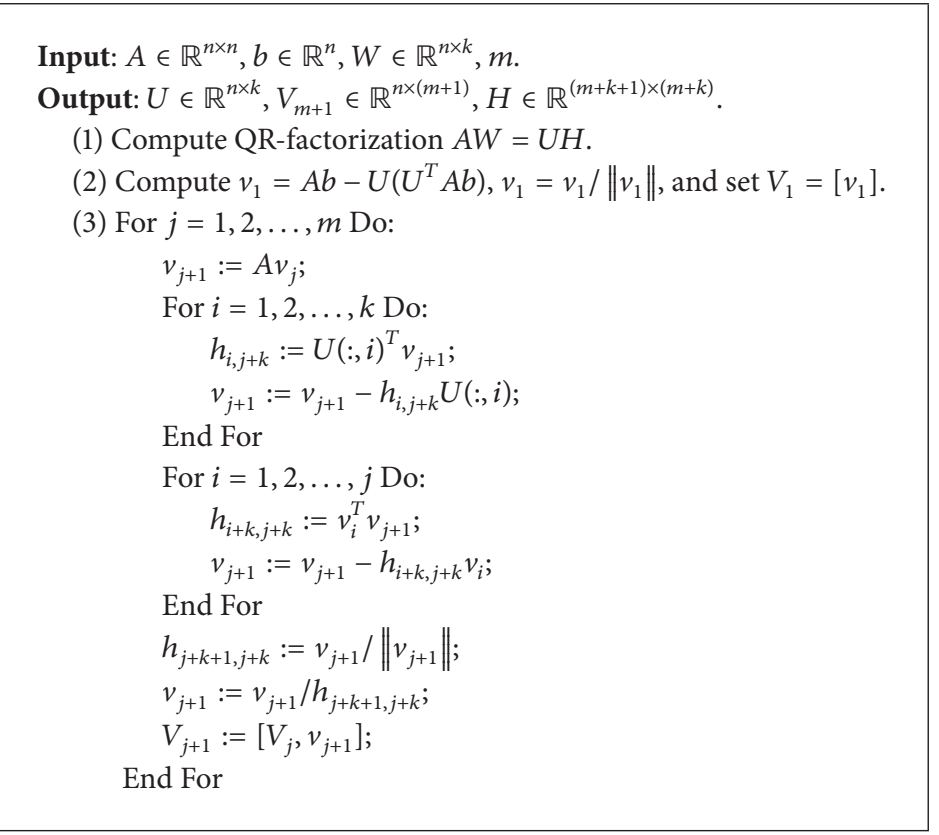

Algorithm 1: Modified Arnoldi process.

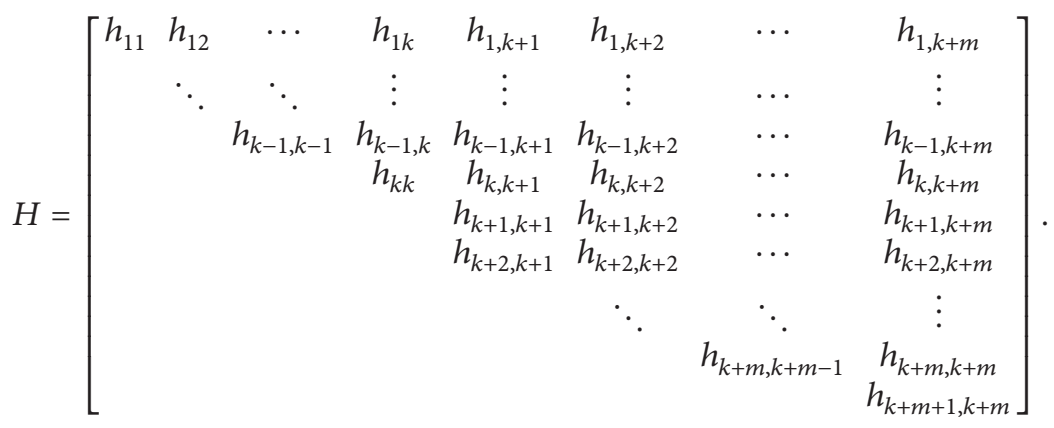

The modified Arnoldi relation (10) is shared by other methods such as GMRES-E [19] and FGMRES [20], which are also augmented type methods.

The modified Arnoldi process is outlined in Algorithm 1. We remark that the modified Arnoldi process in this paper is slightly different from the one used by Morgan [19] to augment the Krylov subspace with some approximate eigenvectors. In his method, the augmenting vectors are put after the Krylov vectors while in Algorithm 1, the augmenting subspace containing a rough approximation solution is included in the projection subspace from the beginning. In general, this can give better results at the start of the regularization method proposed in this paper.

We remark that a loss of orthogonality can occur when the algorithm progresses; see [21]. A remedy is the socalled reorthogonalization where the current vector has to be orthogonalized against previously created vectors. One can choose between a selective reorthogonalization or a full reorthogonalization against all vectors in the current augmented subspace. In this paper we only use the full reorthogonalization. The full reorthogonalization can be done as a classical or modifed Gram-Schmidt orthogonalization; see [21] for details.

We now seek to determine an approximate solution $x_{u, m}$ of (3) in the augmented Krylov subspace $\mathscr{K}_{m}(A, A b)+\mathscr{W}$. After computing the $Q R$ factorization $\left[W, V_{m}\right]=Q R$ with $Q \in$ $\mathbb{R}^{n \times(m+k)}$ having orthonormal columns and $R \in \mathbb{R}^{(m+k) \times(m+k)}$ being an upper triangular matrix, we substitute $x=\left[W, V_{m}\right] y$, $y \in \mathbb{R}^{m+k}$, into (3). It yields the reduced minimization problem

$$
\begin{aligned}
& \min _{y \in \mathbb{R}^{m+k}}\left\{\left\|A\left[W, V_{m}\right] y-b\right\|^{2}+\frac{1}{\mu}\left\|\left[W, V_{m}\right] y\right\|^{2}\right\} \\
& =\min _{y \in \mathbb{R}^{m+k}}\left\{\left\|\left[U, V_{m+1}\right] H y-b\right\|^{2}+\frac{1}{\mu}\|Q R y\|^{2}\right\}
\end{aligned}
$$




$$
\begin{aligned}
=\min _{y \in \mathbb{R}^{m+k}}\left\{\left\|H y-\left[U, V_{m+1}\right]^{T} b\right\|^{2}\right. \\
\left.+\|(I-P) b\|^{2}+\frac{1}{\mu}\|R y\|^{2}\right\},
\end{aligned}
$$

in which $P=\left[U, V_{m+1}\right]\left[U, V_{m+1}\right]^{T}$ is an orthogonal projector onto $\operatorname{span}\left(\left[U, V_{m+1}\right]\right)$. Obviously, the reduced minimization problem (12) is equivalent to

$$
\begin{aligned}
& \min _{y \in \mathbb{R}^{m+k}}\left\{\left\|H y-\left[U, V_{m+1}\right]^{T} b\right\|^{2}+\frac{1}{\mu}\|R y\|^{2}\right\} \\
& =\min _{y \in \mathbb{R}^{m+k}}\left\|\left[\begin{array}{c}
H \\
\frac{1}{\sqrt{\mu}} R
\end{array}\right] y-\left[\begin{array}{c}
{\left[U, V_{m+1}\right]^{T} b} \\
0
\end{array}\right]\right\|^{2} .
\end{aligned}
$$

The normal equations of the minimization problem (13) is

$$
\left(H^{T} H+\frac{1}{\mu} R^{T} R\right) y=H^{T}\left[U, V_{m+1}\right]^{T} b .
$$

We denote the solution of the minimization problem (13) by $y_{\mu, m}$. Then, from (14) it follows that

$$
y_{\mu, m}=\left(H^{T} H+\frac{1}{\mu} R^{T} R\right)^{-1} H^{T}\left[U, V_{m+1}\right]^{T} b .
$$

The approximate solution of (3) is

$$
x_{\mu, m}=\left[W, V_{m}\right] y_{\mu, m} .
$$

Since the matrix $H^{T} H+(1 / \mu) R^{T} R$ has a larger condition number than the matrix $\left[H^{T},(1 / \sqrt{\mu}) R^{T}\right]^{T}$, we apply the $\mathrm{QR}$ factorization of $\left[H^{T},(1 / \sqrt{\mu}) R^{T}\right]^{T}$ to obtain the solution $y_{\mu, m}$ of (13) instead. The QR factorization of $\left[H^{T},(1 / \sqrt{\mu}) R^{T}\right]^{T}$ can be implemented by a sequence of Givens rotations.

Define

$$
\phi_{m}(\mu)=\left\|b-A x_{\mu, m}\right\|^{2}
$$

Substituting $x_{\mu, m}=\left[W, V_{m}\right] y_{\mu, m}$ into (17) and using the modified Arnoldi decomposition (10) yield

$$
\begin{aligned}
\phi_{m}(\mu) & =\left\|b-A\left[W, V_{m}\right] y_{\mu, m}\right\|^{2} \\
& =\left\|b-\left[U, V_{m+1}\right] H y_{\mu, m}\right\|^{2} \\
& =\left\|\left[U, V_{m+1}\right]^{T} b-H y_{\mu, m}\right\|^{2}+\|(I-P) b\|^{2} .
\end{aligned}
$$

Substituting $y_{\mu, m}$ in (15) into (18), we obtain

$$
\begin{aligned}
\phi_{m}(\mu) & =\left\|\left[I-H\left(H^{T} H+\frac{1}{\mu} R^{T} R\right)^{-1} H^{T}\right]\left[U, V_{m+1}\right]^{T} b\right\|^{2} \\
& +\|(I-P) b\|^{2} .
\end{aligned}
$$

Note that

$$
I-H\left(H^{T} H+\frac{1}{\mu} R^{T} R\right)^{-1} H^{T}=\left[\mu H R^{-1}\left(H R^{-1}\right)^{T}+I\right]^{-1} .
$$

Therefore, the function $\phi_{m}(\mu)$ can be expressed as

$$
\begin{aligned}
\phi_{m}(\mu)= & \left\|\left[\mu H R^{-1}\left(H R^{-1}\right)^{T}+I\right]^{-1}\left[U, V_{m+1}\right]^{T} b\right\|^{2} \\
& +\|(I-P) b\|^{2} .
\end{aligned}
$$

Concerning the properties of $\phi_{m}(\mu)$, we have the following results, which are similar to those of Theorem 2.1 in [11] for the Arnoldi-Tikhonov regularization method.

Theorem 1. The function $\phi_{m}(\mu)$ has the representation

$$
\begin{aligned}
\phi_{m}(\mu)= & b^{T}\left[U, V_{m+1}\right]\left[\mu H R^{-1}\left(H R^{-1}\right)^{T}+I\right]^{-2} \\
& \times\left[U, V_{m+1}\right]^{T} b+\|(I-P) b\|^{2} .
\end{aligned}
$$

Assume that $A b \neq 0$ and $\left(H R^{-1}\right)^{T}\left[U, V_{m+1}\right]^{T} b \neq 0$. Then $\phi_{m}$ is strictly decreasing and convex for $\mu \geq 0$ with $\phi_{m}(0)=\|b\|^{2}$. Moreover, the equation

$$
\phi_{m}(\mu)=\tau
$$

has a unique solution $\mu_{\tau, m}$, such that $0<\mu_{\tau, m}<\infty$, for any $\tau$ with

$$
\left\|P_{\mathcal{N}\left(\left(H R^{-1}\right)^{T}\right)}\left[U, V_{m+1}\right]^{T} b\right\|^{2}+\|(I-P) b\|^{2}<\tau<\|b\|^{2},
$$

where $P_{\mathcal{N}\left(\left(H R^{-1}\right)^{T}\right)}$ denotes the orthogonal projector onto $\mathcal{N}\left(\left(H R^{-1}\right)^{T}\right)$.

Proof. The proof follows the same argument of the proof of Theorem 2.1 in [11] and therefore is omitted.

We easily obtain the following theorem, of which the proof is almost the same as that of Corollary 2.2 in [11].

Theorem 2. Assume that the modified Arnoldi process breaks down at step $j$. Then the sequence $\left\{s_{i}\right\}_{i=0}^{j}$ defined by

$$
\begin{gathered}
s_{0}=\|b\|^{2}, \\
s_{i}=\left\|P_{\mathcal{N}\left(\left(H R^{-1}\right)^{T}\right)}\left[U, V_{m+1}\right]^{T} b\right\|^{2}+\|(I-P) b\|^{2}, \quad 0<i<j, \\
s_{j}=0,
\end{gathered}
$$

is decreasing.

We apply the discrepancy principle to the discrepancy $b-$ $A x_{\mu, m}$ to determine an appropriate regularization parameter $\mu$ so that it satisfies (5). To make the equation

$$
\phi_{m}(\mu)=\eta^{2} \varepsilon^{2}
$$


Input: $A \in \mathbb{R}^{n \times n}, b \in \mathbb{R}^{n}, W \in \mathbb{R}^{n \times k}, \varepsilon, \eta, m_{0}$.

Output: $x_{\mu, m}, \mu_{m}, m=m_{\min }+m_{0}$.

(1) Compute the modified Arnoldi decomposition $A\left[W, V_{m}\right]=\left[U, V_{m+1}\right] H$, with $m=m_{\min }+m_{0}$, where $m_{\min }$ is the smallest number such that $\|(I-P) b\|^{2}<\eta^{2} \varepsilon^{2}$.

(2) Compute the solution $\mu_{m}$ of the equation $\phi_{m}(\mu)=\eta^{2} \varepsilon^{2}$ by Newton's method.

(3) Compute the solution $y_{\mu, m}$ of the least-squares problem (13) and obtain the approximate solution $x_{\mu, m}=\left[W, V_{m}\right] y_{\mu, m}$.

Algorithm 2: Augmented Arnoldi-Tikhonov regularization method.

have a solution, it follows from Theorem 1 that the input parameter $m$ of the modified Arnoldi should be chosen so that $s_{m}<\eta^{2} \varepsilon^{2}$. To simplify the computations, we ignore the first term in $s_{m}$. Then, the smallest iterative step number, denoted by $m_{\min }$, of the modified Arnoldi process is chosen so that

$$
\|(I-P) b\|^{2}<\eta^{2} \varepsilon^{2} .
$$

We can improve the quality of the computed solution by choosing the practical iterative step number $m$ somewhat larger than $m_{\min }$.

After choosing the number $m$ of the modified Arnoldi iterative steps, the regularization parameter $\mu_{m}$ is determined by solving the nonlinear equation $\phi_{m}(\mu)=\eta^{2} \varepsilon^{2}$. Many numerical methods have been proposed for the solution of a nonlinear equation, including Newton's method [22], super-Newton's [23] method, and Halley's method [24]. For the specific nonlinear equation $\phi_{m}(\mu)=\eta^{2} \varepsilon^{2}$, Reichel and Shyshkov proposed a new zero-finder method in their new paper [25]. In this paper, we still make use of Newton's method to obtain the regularization parameter $\mu_{m}$.

In Algorithm 2, we outline the augmented ArnoldiTikhonov regularization method, which is used to solve largescale linear ill-posed systems (1).

Newton's method requires to evaluate $\phi_{m}(\mu)$ and its first derivative with respect to $\mu$ for computing approximations $\mu_{m}^{(j)}$ of $\mu_{m}$ for $j=0,1,2, \ldots$ Let

$$
z_{\mu, m}=\left[\mu H R^{-1}\left(H R^{-1}\right)^{T}+I\right]^{-1}\left[U, V_{m+1}\right]^{T} b ;
$$

that is, $z_{\mu, m}$ satisfies the system of linear equations

$$
\left[\mu H R^{-1}\left(H R^{-1}\right)^{T}+I\right] z=\left[U, V_{m+1}\right]^{T} b .
$$

Note that the above linear system is the normal equations of the least-squares problem

$$
\min _{z \in \mathbb{R}^{m+k+1}}\left\|\left[\begin{array}{c}
\mu^{1 / 2}\left(H R^{-1}\right)^{T} \\
I
\end{array}\right] z-\left[\begin{array}{c}
0 \\
\left(\mathrm{U}, V_{m+1}\right)^{T} b
\end{array}\right]\right\| .
$$

For numerical stability, we compute the vector $z_{\mu, m}$ by solving the least-squares problem. Then, the $\phi_{m}(\mu)$ is evaluated by computing

$$
\phi_{m}(\mu)=z_{\mu, m}^{T} z_{\mu, m}+\|(I-P) b\|^{2} .
$$

It is easy to show that the first derivative of $\phi_{m}^{\prime}(\mu)$ can be written as

$$
\phi_{m}^{\prime}(\mu)=-2 z_{\mu, m}^{T} w_{\mu, m}
$$

where

$$
w_{\mu, m}=\left[\mu H R^{-1}\left(H R^{-1}\right)^{T}+I\right]^{-1} H R^{-1}\left(H R^{-1}\right)^{T} z_{\mu, m} .
$$

Hence, we may compute $w_{\mu, m}$ by solving a least-squares problem analogous to the above with the vector $\left[U, V_{m+1}\right]^{T} b$ replaced by $H R^{-1}\left(H R^{-1}\right)^{T} z_{\mu, m}$.

The algorithm for implementing the Newton iteration for solving the nonlinear equation $\phi_{m}(\mu)=\eta^{2} \varepsilon^{2}$ is presented in Algorithm 3.

Let $x_{m}$ be the $m$ th iterate produced by the augmented RRGMRES in [16]. Then, $x_{m}$ satisfies

$$
\begin{aligned}
\left\|A x_{m}-b\right\|^{2}= & \min _{x \in \mathscr{K}_{m}(A, A b)+\mathscr{W}}\|A x-b\|^{2} \\
= & \min _{y \in \mathbb{R}^{m+k}}\left\|A\left[W, V_{m}\right] y-b\right\|^{2} \\
= & \min _{y \in \mathbb{R}^{m+k}}\left\|\left[U, V_{m+1}\right] H y-b\right\|^{2} \\
= & \min _{y \in \mathbb{R}^{m+k}}\left\|H y-\left[U, V_{m+1}\right]^{T} b\right\|^{2} \\
& +\|(I-P) b\|^{2} .
\end{aligned}
$$

As $\mu \rightarrow \infty$, the reduced minimization problem (12) is the same as the above minimization problem, which shows that

$$
x_{m}=\lim _{\mu \rightarrow \infty} x_{\mu, m} .
$$

Therefore, we obtain the following result.

Theorem 3. Let $x_{m}$ be the mth iterate determined by augmented RRGMRES applied to (1) with initial iterate $x_{0}=0$. Then

$$
\left\|A x_{m}-b\right\|^{2}=\phi_{m}(\infty) .
$$

\section{Numerical Experiments}

In this section, we present some numerical examples to illustrate the performance of the augmented ArnoldiTikhonov regularization method for the solution of largescale linear ill-posed systems. We compare the augmented 
(1) Set $\mu_{0}=0$ and $i=0$.

(2) Solve the least-squares problem

$\min _{z \in \mathbb{R}^{m+k+1}}\left\|\left[\begin{array}{c}\mu_{i}^{1 / 2}\left(H R^{-1}\right)^{T} \\ I\end{array}\right] z-\left[\begin{array}{c}0 \\ \left(U, V_{m+1}\right)^{T} b\end{array}\right]\right\|$

(3) Compute $\phi_{m}\left(\mu_{i}\right)=z_{\mu_{i}, m}^{T} z_{\mu_{i}, m}+\|(I-P) b\|^{2}$.

(4) Solve the least-squares problem

to obtain $w_{\mu_{i}, m}$.

$$
\min _{z \in \mathbb{R}^{m+k+1}}\left\|\left[\begin{array}{c}
\mu_{i}^{1 / 2}\left(H R^{-1}\right)^{T} \\
I
\end{array}\right] z-\left[\begin{array}{c}
0 \\
H R^{-1}\left(H R^{-1}\right)^{T} z_{\mu_{i}, m}
\end{array}\right]\right\|
$$

(5) Compute $\phi_{m}^{\prime}\left(\mu_{i}\right)=-2 z_{\mu_{i}, m}^{T} w_{\mu_{i}, m}$.

(6) Compute the new approximation

$$
\mu_{i+1}=\mu_{i}-\frac{\phi_{m}\left(\mu_{i}\right)-\eta^{2} \varepsilon^{2}}{\phi_{m}^{\prime}\left(\mu_{i}\right)} .
$$

(7) If $\left|\mu_{i+1}-\mu_{i}\right|<10^{-6}$, stop; else $\mu_{i}:=\mu_{i+1}, i:=i+1$, and go to 2 .

Algorithm 3: Newton's method for $\phi_{m}(\mu)=\eta^{2} \varepsilon^{2}$.

Arnoldi-Tikhonov regularization method implemented by Algorithm 2 to the Arnoldi-Tikhonov regularization method proposed in [11]. The Arnoldi-Tikhonov regularization method is denoted by ATRM while the augmented ArnoldiTikhonov regularization method is denoted by AATRM. In all the following tables, we denote by MV the number of matrix-vector products and by RERR the relative error $\left\|x_{\mu, m}-\widehat{x}\right\| /\|\widehat{x}\|$, where $\widehat{x}$ is the exact solution of the linear error-free system of (2). Note that the number of matrixvector products in Algorithm 2 is $k+m_{\min }+m_{0}$. In all the examples, $\eta=1.01$.

All the numerical experiments are performed in Matlab on a PC with the usual double precision, where the floating point relative accuracy is $2.22 \cdot 10^{-16}$.

Example 4. The first example considered is the Fredholm integral equation of the first kind, which takes the generic form

$$
\int_{0}^{1} k(s, t) x(t) d t=b(s), \quad 0 \leq s \leq 1 .
$$

Here, both the kernel $k(s, t)$ and the right-hand side $b(s)$ are known functions, while $x(t)$ is the unknown function. For test, the kernel $k(s, t)$ and the right-hand side $b(s)$ are chosen as

$$
k(s, t)=\left\{\begin{array}{ll}
s(t-1), & s<t, \\
t(s-1), & s \geq t,
\end{array} \quad b(s)=\frac{s^{3}-s}{6} .\right.
$$

With this choice, the exact solution of (37) is $t$. We use the Matlab program deriv2 from the regularization package [26] to discretize the integral equation (37) and to generate a system of linear equations (2) with the coefficient matrix $A \in$ $\mathbb{R}^{200 \times 200}$ and the solution $\hat{x} \in \mathbb{R}^{200}$. The condition number of $A$ is $4.9 \cdot 10^{4}$. The right-hand side $b$ is given by $b=A \widehat{x}+e$, where the elements of the error vector $e$ are generated from normal distribution with mean zero and the norm of $e$ is $10^{-2} \cdot\|A \widehat{x}\|$. The augmentation subspace is one-dimensional
TABLE 1: Computational results of Example 4.

\begin{tabular}{lccc}
\hline Method & $m_{0}$ & MV & RERR \\
\hline AATRM & 0 & 2 & $1.95 \cdot 10^{-2}$ \\
AATRM & 1 & 3 & $1.17 \cdot 10^{-2}$ \\
AATRM & 2 & 4 & $6.25 \cdot 10^{-2}$ \\
AATRM & 3 & 5 & $1.19 \cdot 10^{-1}$ \\
ATRM & 0 & 4 & $3.14 \cdot 10^{-1}$ \\
ATRM & 1 & 5 & $2.94 \cdot 10^{-1}$ \\
ATRM & 2 & 6 & $2.87 \cdot 10^{-1}$ \\
ATRM & 3 & 7 & $2.89 \cdot 10^{-1}$ \\
\hline
\end{tabular}

and is spanned by the vector $w=[1,2, \ldots, 200]^{T}$. Numerical results for the example are reported in Table 1 for several choice of the number $m_{0}$ of additional iterations.

From Table 1, we can see that for $0 \leq m_{0} \leq 3$, AATRM has smaller relative errors than ATRM, and the smallest relative error is given by AATRM with $m_{0}=1$. The exact solution $\widehat{x}$ and the approximate solutions generated by AATRM and ARTM with $m_{0}=1$ are depicted in Figure 1 .

Example 5. This example comes again from the regularization package [26] and is the inversion of Laplace transform

$$
\int_{0}^{\infty} e^{-s t} x(s) d s=b(t), \quad t \geq 1
$$

where the right-hand side $b(t)$ and the exact solution $x(t)$ are given by

$$
b(t)=\frac{1}{t+1 / 2}, \quad x(t)=e^{-t / 2} .
$$

The system of linear equations (2) with the coefficient matrix $A \in \mathbb{R}^{250 \times 250}$ and the solution $\hat{x} \in \mathbb{R}^{250}$ is obtained by using the Matlab program ilaplace from the regularization package [26]. In the same way as Example 4, we construct 


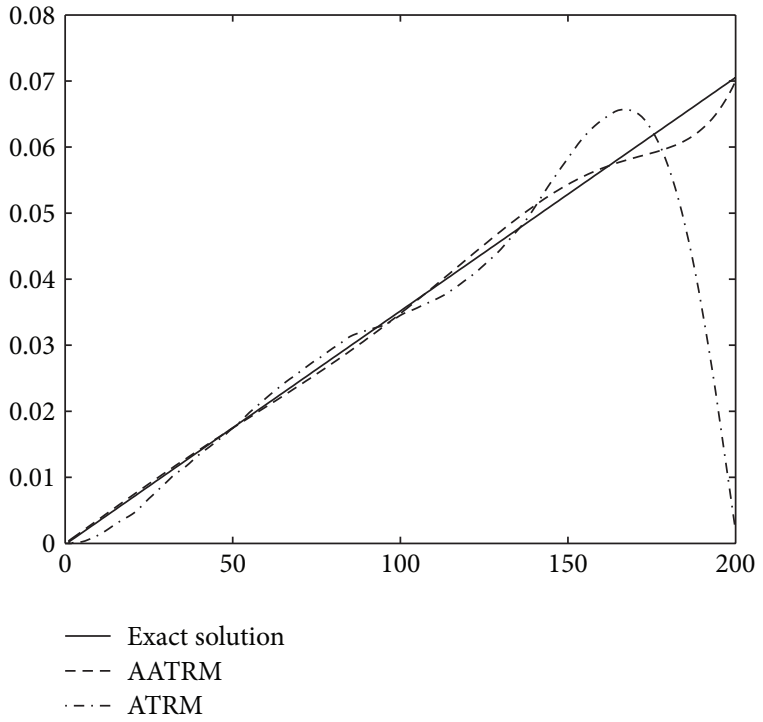

FIGURE 1: Example 4. Exact solution $\widehat{x}$; approximation solutions with $m_{0}=1$.

TABLE 2: Computational results of Example 5.

\begin{tabular}{lccc}
\hline Method & $m_{0}$ & MV & RERR \\
\hline AATRM & 1 & 5 & $3.3 \cdot 10^{-1}$ \\
AATRM & 2 & 6 & $9.1 \cdot 10^{-2}$ \\
AATRM & 3 & 7 & $2.7 \cdot 10^{-1}$ \\
ATRM & 1 & 6 & $5.7 \cdot 10^{-1}$ \\
ATRM & 2 & 7 & $3.1 \cdot 10^{-1}$ \\
ATRM & 3 & 8 & $4.5 \cdot 10^{-1}$ \\
\hline
\end{tabular}

TABle 3: Computational results of Example 6.

\begin{tabular}{lccc}
\hline Method & $m_{0}$ & MV & RERR \\
\hline AATRM & 1 & 10 & $2.2 \cdot 10^{-1}$ \\
AATRM & 2 & 11 & $1.1 \cdot 10^{-1}$ \\
AATRM & 3 & 12 & $2.1 \cdot 10^{-1}$ \\
ATRM & 1 & 10 & $4.9 \cdot 10^{-1}$ \\
ATRM & 2 & 11 & $1.2 \cdot 10^{-1}$ \\
ATRM & 3 & 12 & $2.3 \cdot 10^{-1}$ \\
\hline
\end{tabular}

the right-hand side $b$ of the system of linear equations (1). The augmentation subspace is also one-dimensional and is spanned by the vector $w=[1,1 / 2, \ldots, 1 / 250]^{T}$. Numerical results for the example are reported in Table 2 for $m_{0}=1,2,3$. Table 2 shows that AATRM with $m_{0}=2$ has the smallest relative error, and AARTM works slightly better than ATRM for this problem.

Example 6. This example considered here is the same as Example 5 except that the right-hand side $b(t)$ and the exact solution $x(t)$ are given by

$$
b(t)=\frac{2}{(t+1 / 2)^{3}}, \quad x(t)=t^{2} e^{-t / 2} .
$$

By using the same Matlab program as Example 5, we generate a system with $A \in \mathbb{R}^{1000 \times 1000}$. The augmentation subspace is one-dimensional and is spanned by the vector $w=$ $[1,1, \ldots, 1]^{T}$. In Table 3 , we report numerical results for $m_{0}=$ $1,2,3$.

We observe from Table 3 that for this example AATRM has almost the same relative errors as ATRM, and the approximate solution can be slightly improved by the augmentation space spanned by $w=[1,1, \ldots, 1]^{T}$.

\section{Conclusions}

In this paper we propose an iterative method for solving large-scale linear ill-posed systems. The method is based on the Tikhonov regularization technique and the augmented Arnoldi technique. The augmentation subspace is a usersupplied low-dimensional subspace, which should contain a rough approximation of the desired solution. Numerical experiments show that the augmented method is effective for some practical problems.

\section{Acknowledgments}

Yiqin Lin is supported by the National Natural Science Foundation of China under Grant 10801048, the Natural Science Foundation of Hunan Province under Grant 11JJ4009, the Scientific Research Foundation of Education Bureau of Hunan Province for Outstanding Young Scholars in University under Grant 10B038, the Science and Technology Planning Project of Hunan Province under Grant 2010JT4042, and the Chinese Postdoctoral Science Foundation under Grant 2012M511386. Liang Bao is supported by the National Natural Science Foundation of China under Grants 10926150 and 11101149 and the Fundamental Research Funds for the Central Universities.

\section{References}

[1] H. W. Engl, M. Hanke, and A. Neubauer, Regularization of Inverse Problems, vol. 375 of Mathematics and Its Applications, Kluwer Academic, Dordrecht, The Netherlands, 1996.

[2] A. N. Tikhonov and V. Y. Arsenin, Solutions of Ill-Posed Problems, John Wiley \& Sons, New York, NY, USA, 1977.

[3] A. N. Tikhonov, A. V. Goncharsky, V. V. Stepanov, and A. G. Yagola, Numerical Methods for the Solution of Ill-Posed Problems, vol. 328 of Mathematics and Its Applications, Kluwer Academic, Dordrecht, The Netherlands, 1995.

[4] P. C. Hansen, Rank-Deficient and Discrete Ill-Posed Problems, SIAM Monographs on Mathematical Modeling and Computation, SIAM, Philadelphia, Pa, USA, 1998.

[5] G. H. Golub and C. F. van Loan, Matrix Computations, Johns Hopkins Studies in the Mathematical Sciences, Johns Hopkins University Press, Baltimore, Md, USA, 3rd edition, 1996.

[6] Å. Björck, Numerical Methods for Least Squares Problems, SIAM, Philadelphia, Pa, USA, 1996.

[7] Å. Björck, "A bidiagonalization algorithm for solving large and sparse ill-posed systems of linear equations," BIT: Numerical Mathematics, vol. 28, no. 3, pp. 659-670, 1988.

[8] D. Calvetti, S. Morigi, L. Reichel, and F. Sgallari, “Tikhonov regularization and the $L$-curve for large discrete ill-posed 
problems," Journal of Computational and Applied Mathematics, vol. 123, no. 1-2, pp. 423-446, 2000.

[9] D. Calvetti and L. Reichel, "Tikhonov regularization of large linear problems," BIT: Numerical Mathematics, vol. 43, no. 2, pp. 263-283, 2003.

[10] D. P. O'Leary and J. A. Simmons, "A bidiagonalizationregularization procedure for large scale discretizations of illposed problems," SIAM Journal on Scientific and Statistical Computing, vol. 2, no. 4, pp. 474-489, 1981.

[11] B. Lewis and L. Reichel, "Arnoldi-Tikhonov regularization methods," Journal of Computational and Applied Mathematics, vol. 226, no. 1, pp. 92-102, 2009.

[12] T. F. Chan and K. R. Jackson, "Nonlinearly preconditioned Krylov subspace methods for discrete Newton algorithms," SIAM Journal on Scientific and Statistical Computing, vol. 5, no. 3, pp. 533-542, 1984.

[13] L. Reichel, F. Sgallari, and Q. Ye, “Tikhonov regularization based on generalized Krylov subspace methods," Applied Numerical Mathematics, vol. 62, no. 9, pp. 1215-1228, 2012.

[14] D. Calvetti, B. Lewis, and L. Reichel, "On the choice of subspace for iterative methods for linear discrete ill-posed problems," International Journal of Applied Mathematics and Computer Science, vol. 11, no. 5, pp. 1069-1092, 2001, Numerical analysis and systems theory (Perpignan, 2000).

[15] L. Reichel and Q. Ye, "Breakdown-free GMRES for singular systems," SIAM Journal on Matrix Analysis and Applications, vol. 26, no. 4, pp. 1001-1021, 2005.

[16] J. Baglama and L. Reichel, "Augmented GMRES-type methods," Numerical Linear Algebra with Applications, vol. 14, no. 4, pp. 337-350, 2007.

[17] K. Morikuni, L. Reichel, and K. Hayami, "FGMRES for linear discrete problems," Tech. Rep., NII, 2012.

[18] D. Calvetti, B. Lewis, and L. Reichel, "On the regularizing properties of the GMRES method," Numerische Mathematik, vol. 91, no. 4, pp. 605-625, 2002.

[19] R. B. Morgan, "A restarted GMRES method augmented with eigenvectors," SIAM Journal on Matrix Analysis and Applications, vol. 16, no. 4, pp. 1154-1171, 1995.

[20] Y. Saad, "A flexible inner-outer preconditioned GMRES algorithm," SIAM Journal on Scientific Computing, vol. 14, no. 2, pp. 461-469, 1993.

[21] B. N. Parlett, The Symmetric Eigenvalue Problem, vol. 20 of Classics in Applied Mathematics, SIAM, Philadelphia, Pa, USA, 1998.

[22] C. T. Kelley, Solving Nonlinear Equations with Newton's Method, vol. 1 of Fundamentals of Algorithms, SIAM, Philadelphia, Pa, USA, 2003.

[23] J. A. Ezquerro and M. A. Hernández, "On a convex acceleration of Newton's method," Journal of Optimization Theory and Applications, vol. 100, no. 2, pp. 311-326, 1999.

[24] W. Gander, “On Halley's iteration method," The American Mathematical Monthly, vol. 92, no. 2, pp. 131-134, 1985.

[25] L. Reichel and A. Shyshkov, "A new zero-finder for Tikhonov regularization," BIT: Numerical Mathematics, vol. 48, no. 3, pp. 627-643, 2008.

[26] P. C. Hansen, "Regularization tools: a Matlab package for analysis and solution of discrete ill-posed problems," Numerical Algorithms, vol. 6, no. 1-2, pp. 1-35, 1994. 


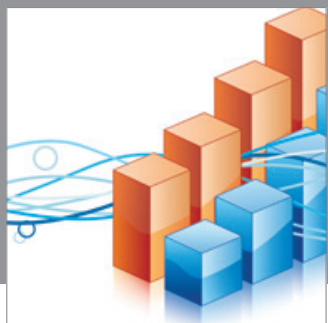

Advances in

Operations Research

mansans

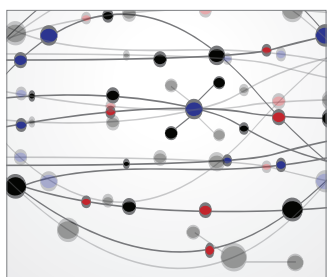

The Scientific World Journal
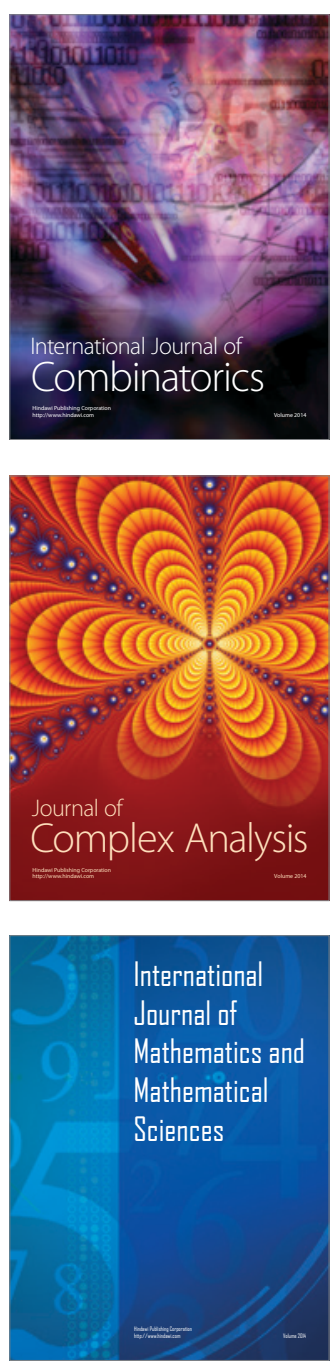
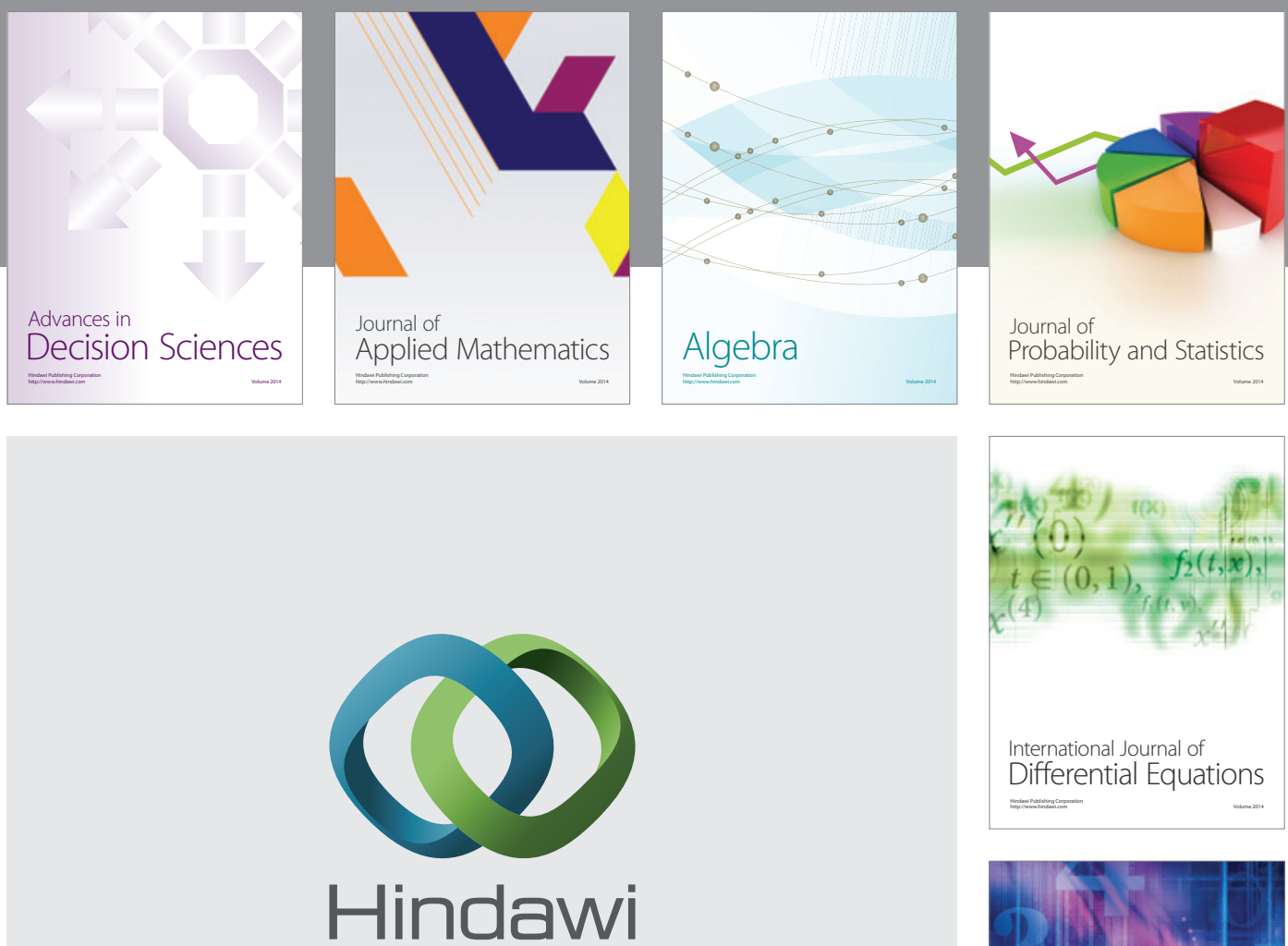

Submit your manuscripts at http://www.hindawi.com
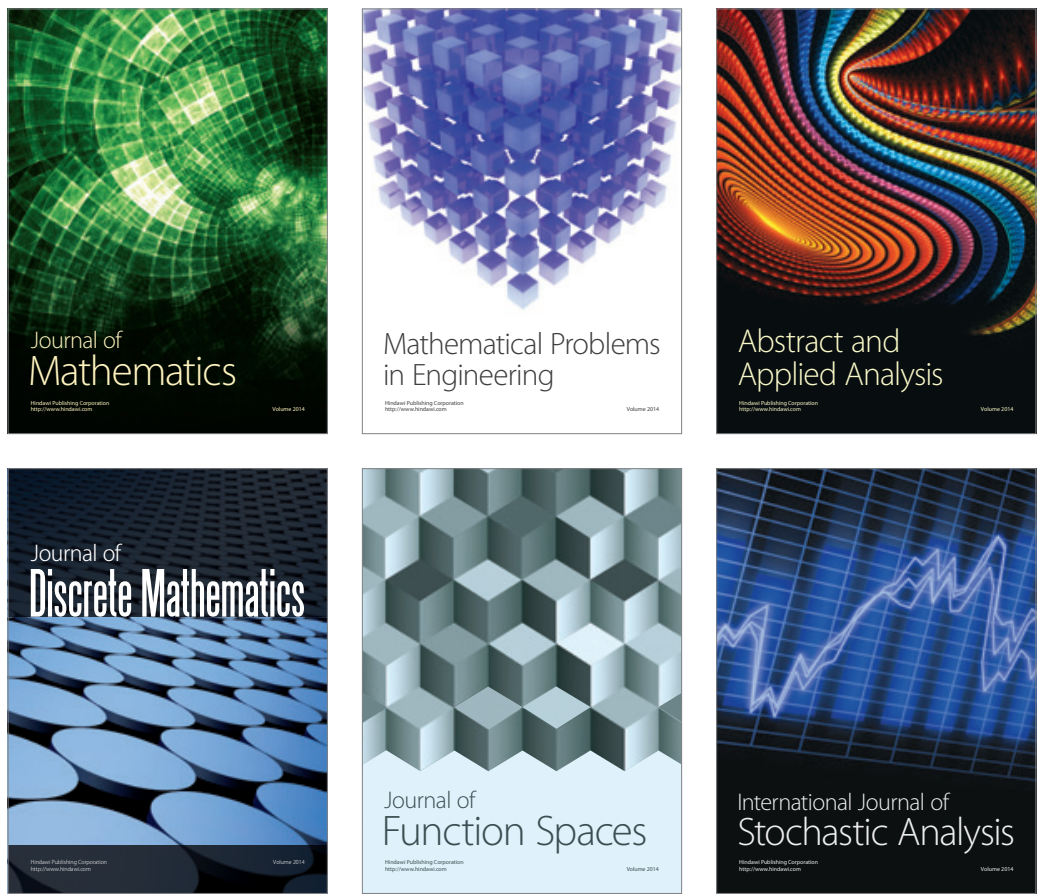

Journal of

Function Spaces

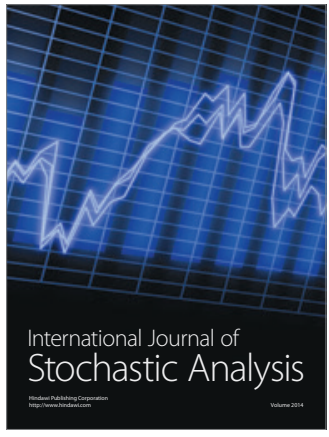

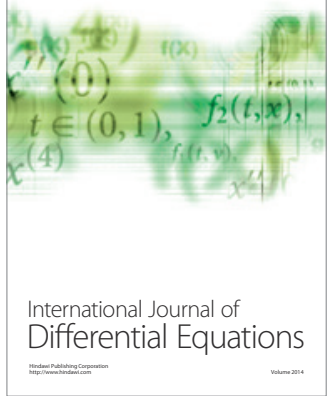
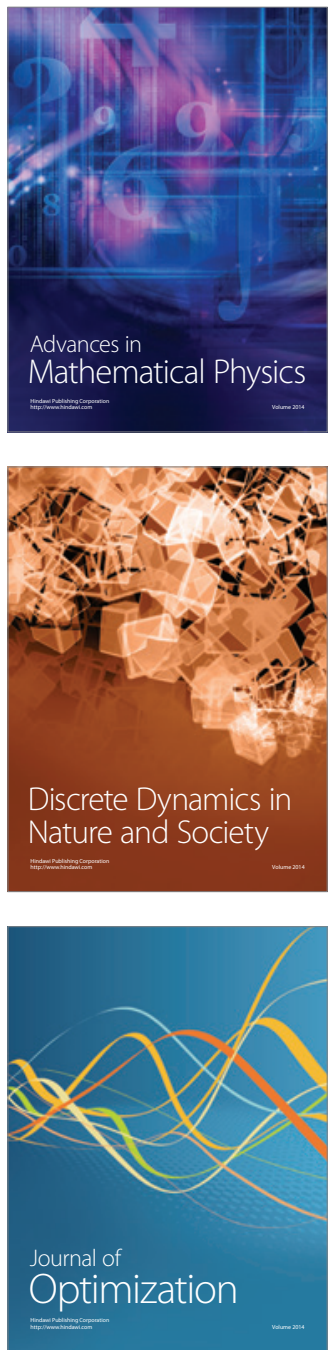